\title{
Neutrophil gelatinase-associated lipocalin immunoexpression in renal tumors: Correlation with histotype and histological grade
}

\author{
VALERIA BARRESI ${ }^{1}$, ANTONIO IENI $^{1}$, DAVIDE BOLIGNANO ${ }^{2}$, CARLO MAGNO $^{1}$, \\ MICHELE BUEMI $^{2}$ and GAETANO BARRESI ${ }^{1}$
}

Departments of ${ }^{1}$ Human Pathology and ${ }^{2}$ Internal Medicine, University of Messina, Messina, Italy

Received November 4, 2009; Accepted January 21, 2010

DOI: $10.3892 /$ or_00000860

\begin{abstract}
Neutrophil gelatinase-associated lipocalin (NGAL) is a $25 \mathrm{kDa}$ protein with roles in iron trafficking as well as in carcinogenesis and progression of several human neoplasias. Although the renal proximal tubule represents a major source of NGAL synthesis under various injurious stimuli to the kidney, NGAL expression has been rarely evaluated in renal tumors up to now. In view of this, in the present study we analyzed the expression of this protein in renal tumors of different histotype and grade so as to evaluate whether a role for NGAL might be also proposed in the carcinogenesis of these neoplasms. NGAL immunoexpression was analyzed in 30 surgically resected renal tumors [18 clear cell, 5 papillary and 3 chromophobe renal cell carcinomas (RCCs), 2 urothelial carcinomas and 2 oncocytomas] and in the peritoneal metastasis of a clear cell RCC. A variable NGAL immunoexpression was found in $28 / 30$ cases. High NGAL expression was significantly associated with the papillary and chromphobe histotypes $(\mathrm{P}=0.016)$ and with a higher histological grade of clear cell and papillary RCC ( $\mathrm{P}=0.004)$. Moreover, NGAL expression was retained in the peritoneal metastasis of clear cell RCC. Our findings demonstrate that NGAL is expressed in several histotypes of renal tumors. Its highest expression in the papillary and chromophobe histotypes might be related to a higher need in iron uptake, which could be exploited in anti-cancer therapies with iron chelators against these neoplasias. Further studies are required to investigate the potential diagnostic utility of NGAL in the early diagnosis of metastatic progression of RCC.
\end{abstract}

Correspondence to: Dr Valeria Barresi, Dipartimento di Patologia Umana, Policlinico, University G. Martino, Pad D, Via Consolare Valeria, 98125 Messina, Italy

E-mail: vbarresi@unime.it

Key words: neutrophil gelatinase-associated lipocalin, renal tumors, iron, Fuhrman grade

\section{Introduction}

Neutrophil gelatinase-associated lipocalin (NGAL) is also known as NRL (neu-related lipocalin), oncogene 24p3, uterocalin and lipocalin 2 (LCN2). It is a $25 \mathrm{kDa}$ protein, which is stored in the granules of human neutrophils (1) and which belongs to the lipocalin family that comprises more than 50 members all of which are characterized by the ability to bind and transport small lipophilic substances (2). NGAL has been shown to participate in iron trafficking (3). Indeed, this protein captures and transports the iron particles to the inner cell after interaction with specific membrane receptors (24p3, megalin), thus increasing the cytoplasmic levels of this mineral (4). Its role in the iron delivery into the cells underlies the multiple effects attributed to NGAL. Released by activated neutrophils, this protein participates in the iron depletion strategy exploited in the immune defense against bacterial pathogens (5). In addition, NGAL seems to be involved in the growth, development and differentiation of several human tissues, as early as in the embryo, through its regulation of iron responsive-genes which are important in the differentiation of primordial cells $(6,7)$.

Since NGAL synthesis is induced by factors promoting the development of neoplasias $(2,8,9)$, this protein has been suggested to play a key role also in the carcinogenesis and progression of human tumors (10), similarly to other lipocalins (2). According to a recent theory, the increased NGAL synthesis would favor iron uptake from extracellular space within the malignant cells, a fundamental process for the maintenance of neoplastic cells multiplication $(11,12)$. Moreover, NGAL is known to complex with matrix metalloproteinase-9, which is a promoter of carcinogenesis by degrading the basement membranes and extracellular matrix, liberating vascular endothelial growth factor (VEGF), and thus enabling angiogenesis, invasion and metastasis $(13,15,16)$. In line with a pro-tumorigenic function of this protein, NGAL overexpression has been found in several tumors, including breast $(17,18)$, thyroid (12) and colorectal carcinomas $(19,20)$, primary gastric adenocarcinomas $(21)$, esophageal squamous cell carcinomas (22) and human pancreatic cancers (23).

Although NGAL is known to be expressed in the renal proximal tubule epithelial cells (24) and massively released 
Table I. Clinicopathological features and NGAL immunohistochemical data relative to the 30 analyzed renal tumors.

\begin{tabular}{|c|c|c|c|c|c|c|c|c|c|c|}
\hline Case & Gender & Age & $\begin{array}{c}\text { Size } \\
(\mathrm{cms})\end{array}$ & T stage & Histotype & Grade & $\begin{array}{c}\text { NGAL } \\
\text { ASP }\end{array}$ & $\begin{array}{c}\text { NGAL } \\
\text { IS }\end{array}$ & $\begin{array}{l}\text { NGAL ID } \\
\text { score }\end{array}$ & $\begin{array}{l}\text { Serum iron } \\
\quad(\mu \mathrm{g} / \mathrm{dl})\end{array}$ \\
\hline 1 & $\mathrm{~F}$ & 58 & 2.5 & pT3 & Clear cell & G1 & 0 & 1 & 0 & 65 \\
\hline 2 & $\mathrm{~F}$ & 84 & 7 & pT3 & Clear cell & G3 & 4 & 2 & 8 & 30 \\
\hline 3 & M & 77 & 6 & pT1 & Papillary & G2 & 2 & 2 & 4 & 46 \\
\hline 4 & $\mathrm{~F}$ & 60 & 8.5 & pT2 & Clear cell & $\mathrm{G} 2$ & 4 & 1 & 4 & 43 \\
\hline 5 & M & 77 & 3 & pT1 & Clear cell & G1 & 3 & 1 & 3 & 125 \\
\hline 6 & M & 60 & 4 & pT1 & Clear cell & G3 & 3 & 2 & 6 & 61 \\
\hline 7 & $\mathrm{~F}$ & 70 & 3 & pT1 & Papillary & G3 & 4 & 3 & 12 & 89 \\
\hline 8 & $\mathrm{~F}$ & 40 & 9 & pT4 & Clear cell & G2 & 4 & 2 & 8 & 90 \\
\hline 9 & $\mathrm{~F}$ & 60 & 8 & pT2 & Papillary & G3 & 4 & 2 & 8 & 32 \\
\hline 10 & $\mathrm{~F}$ & 75 & 9 & pT3 & Sarcomatoid & G2 & 0 & 1 & 0 & 85 \\
\hline 11 & $\mathrm{~F}$ & 78 & 4.4 & pT1 & Clear cell & $\mathrm{G} 2$ & 2 & 2 & 4 & 78 \\
\hline 12 & M & 56 & 6.3 & pT1 & Clear cell & $\mathrm{G} 2$ & 2 & 2 & 4 & 38 \\
\hline 13 & M & 62 & 8 & pT2 & Clear cell & G1 & 1 & 1 & 1 & 100 \\
\hline 14 & M & 67 & 8 & pT2 & Clear cell & $\mathrm{G} 2$ & 2 & 1 & 2 & 118 \\
\hline 15 & $\mathrm{~F}$ & 40 & 4 & pT1 & Oncocytoma & & 1 & 2 & 2 & 24 \\
\hline 16 & M & 59 & 5 & $\mathrm{pT} 1 \mathrm{~b}$ & Clear cell & G1 & 1 & 1 & 1 & 65 \\
\hline 17 & M & 70 & 6.7 & $\mathrm{pT} 1 \mathrm{~b}$ & Clear cell & G1 & 2 & 2 & 4 & 78 \\
\hline 18 & M & 37 & 5 & $\mathrm{pT} 1$ & Clear cell & $\mathrm{G} 2$ & 2 & 3 & 6 & 100 \\
\hline 19 & M & 69 & 3 & pT2 & Clear cell & G1 & 1 & 1 & 1 & 34 \\
\hline 20 & $\mathrm{~F}$ & 61 & 6 & pT1 & Oncocytoma & & 2 & 1 & 2 & 83 \\
\hline 21 & M & 75 & 5 & pT1 & Clear cell & G1 & 1 & 1 & 1 & 72 \\
\hline 22 & M & 58 & 2.6 & pT1 & Urothelial & & 1 & 1 & 1 & 45 \\
\hline 23 & M & 36 & 6 & pT1 & Clear cell & G2 & 2 & 1 & 2 & 33 \\
\hline 24 & $\mathrm{~F}$ & 65 & 6 & pT1 & Papillary & G3 & 3 & 2 & 6 & 21 \\
\hline 25 & M & 56 & 8 & pT2 & Papillary & G3 & 3 & 2 & 6 & 71 \\
\hline 26 & $\mathrm{~F}$ & 62 & 4 & pT1 & Clear cell & G1 & 2 & 1 & 2 & 31 \\
\hline 27 & $\mathrm{~F}$ & 49 & 8 & $\mathrm{pT} 2$ & Urothelial & & 1 & 1 & 1 & 95 \\
\hline 28 & $\mathrm{~F}$ & 52 & 5 & pT1b & Chromophobe & & 4 & 2 & 8 & 45 \\
\hline 29 & M & 64 & 4.5 & pT1 & Chromophobe & & 4 & 2 & 8 & 35 \\
\hline 30 & M & 60 & 7 & pT3a & Chromophobe & & 4 & 2 & 8 & 65 \\
\hline
\end{tabular}

ID, intensity distribution; ASP, area of staining positivity; IS, intensity of staining; F, female; M, male.

from these cells after various injurious stimuli to the kidney $(25,26)$, its expression in renal cell carcinomas has been evaluated only in one study up to now (24).

In view of this, in the present study we assessed NGAL expression in different types of renal tumors and analyzed its correlations with clinicopathological parameters, including the histological grade and histotype, so as to investigate whether a role for NGAL might be also hypothesized in renal tumors.

\section{Materials and methods}

Thirty cases of surgically resected sporadic renal tumors, obtained from 14 female and 16 male patients (age range: 36-84 years; mean age 61.57 years), were taken from the files of the Department of Human Pathology of the University of Messina, Italy. All cases contained non-neoplastic and non-infiltrated adjacent renal parenchyma. The histological diagnosis was established according to the Heidelberg Classification of Renal Cell Tumors (27). The cohort of the study comprised: 18 clear cell renal cell carcinomas (RCCs) (one of which displayed sarcomatoid differentiation), 5 papillary RCCs, 3 chromophobe RCCs, 2 urothelial carcinomas and 2 oncocytomas. Clear cell and papillary RCRs were graded according to the Fuhrman system (28). For each tumor, the size and $\mathrm{T}$ stage were taken into consideration. $\mathrm{T}$ stage was defined on the basis of the TNM Classification System (29). A specimen representing the peritoneal metastasis of one clear cell RCC (case no. 8) was also submitted to immunohistochemical analysis. The serum iron level of each patient was known; values between 50 and $180 \mu \mathrm{g} / \mathrm{dl}$ were considered as normal.

Immunohistochemistry. All specimens were fixed in $10 \%$ neutral formalin for $24 \mathrm{~h}$ at room temperature, embedded in paraffin at $55^{\circ} \mathrm{C}$ and cut into parallel consecutive $4 \mu \mathrm{m}$ 

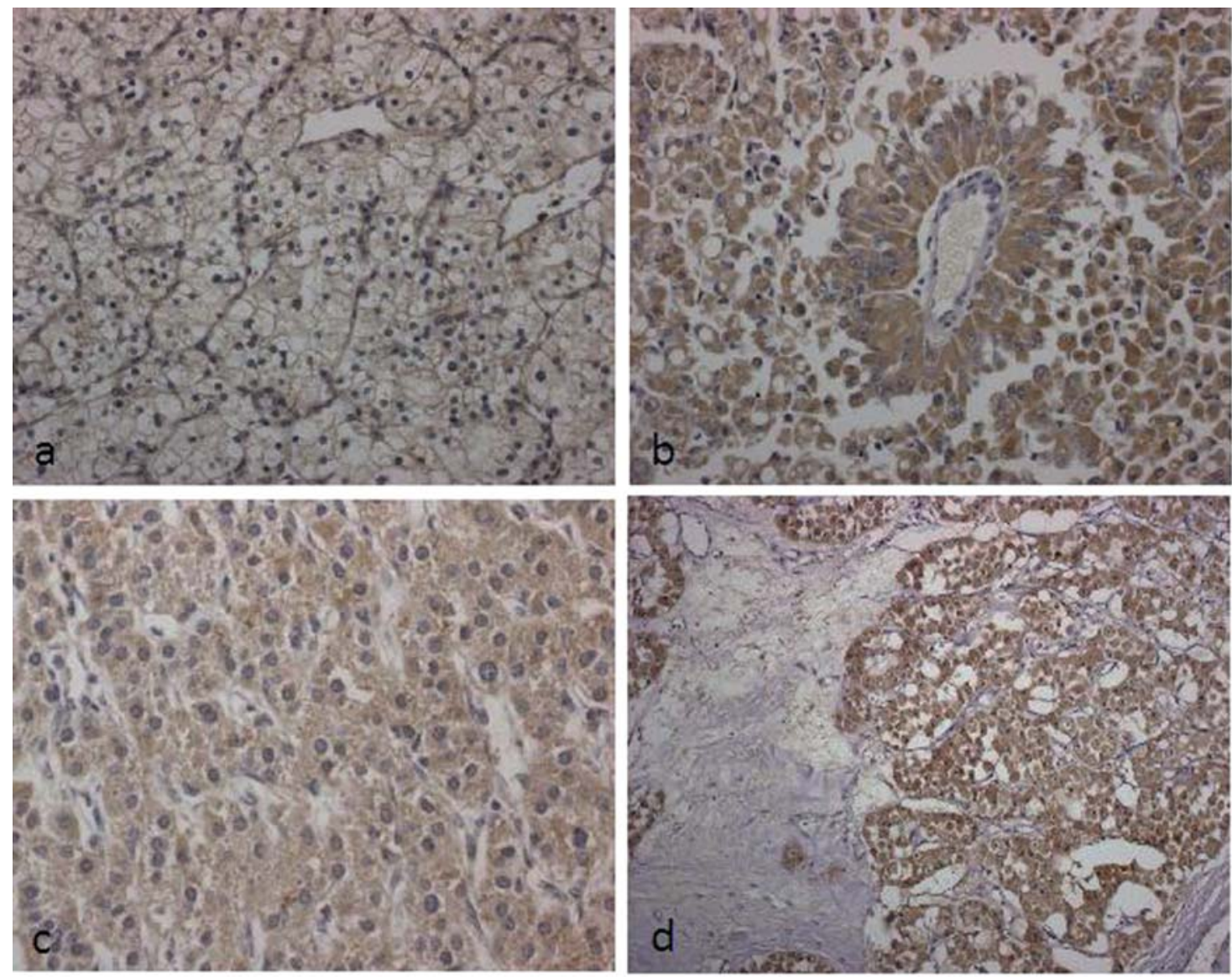

Figure 1 (a) Membranous staining in the neoplastic cells of a clear cell RCC (NGAL stain; original magnification, x200). NGAL immunoexpression in (b) papillary RCC (NGAL stain; original magnification, x200), (c) oncocytoma (NGAL stain; original magnification, x200) and (d) chromophobe RCC (NGAL stain; original magnification, $\mathrm{x} 100)$.

sections for the subsequent immunohistochemical study. Briefly, the endogenous peroxidase activity was blocked with $0.1 \% \mathrm{H}_{2} \mathrm{O}_{2}$ in methanol for $20 \mathrm{~min}$; then, normal sheep serum was applied for $30 \mathrm{~min}$ to prevent unspecific adherence of serum proteins. Sections were successively incubated at $4^{\circ} \mathrm{C}$ overnight with the primary antibody against NGAL (Santa Cruz Biotechnology, Santa Cruz, CA; w.d. 1:100). The bound primary antibody was visualized by the envision peroxidase detection system. To reveal the immunostaining, the sections were incubated in darkness for 10 min with 3,3' diaminobenzidine tetrahydrochloride (Sigma Chemical Co., St. Louis, MO, USA), $100 \mathrm{mg}$ in $200 \mathrm{ml}$ of $0.03 \%$ hydrogen peroxide in phosphate-buffered saline (PBS). Nuclear counterstaining was performed by Mayer's haemalum. Specificity of the binding was assessed by omitting the primary antiserum or replacing it with normal murine serum or phosphate-buffered saline solution (PBS, pH 7.4). Proximal tubules within the normal renal parenchyma adjacent to the tumor were used as normal positive internal controls for the immunoreactions $(24,30)$.

The assessment of immuno-stained sections was performed by two independent pathologists, blinded to the clinicopathological data. NGAL expression was based on the presence of cytoplasmic and membranous staining. The intensity of staining (IS) was graded as 0 , negative; 1 , weak; 2 , moderate; 3 , strong; the area of staining positivity (ASP), recorded as percentage of neoplastic positive cells, was assessed by the following values: $0(\leq 10 \%), 1(11-25 \%), 2(26-50 \%), 3$ $(51-75 \%), 4(>75 \%)$, according to the procedure described by Moniaux et al (31). Then, an intensity-distribution (ID) score was generated for each case by multiplying the values of IS and ASP. Cases displaying an ID score 0 were considered as negative for NGAL.

For the statistical analyses, samples were subdivided into tumors characterized by low (ID score: $<4$ ) and high (ID score: $\geq 4)$ NGAL expression by using the median NGAL ID score (median ID score: 4 ) as the cut-off.

Fisher exact and Chi-square tests were performed to assess the statistical correlations between NGAL expression and various clinicopathological parameters, such as the age and gender of the patients, or the size, the histotype, the histological grade and the T stage of the tumors. Again, Fisher exact test was used to analyze the correlation between NGAL immunoexpression and serum iron.

$\mathrm{P}<0.05$ was considered statistically significant. Data were analysed using the SPSS package version 6.1.3 (SPSS Inc., Chicago, IL, USA).

\section{Results}

NGAL immunostaining was encountered in the normal tubules adjacent to the tumors; in addition, a weak labelling was 
evidenced in the epithelial cells of collector tubules and in the urothelium of pelvis.

The immunohistochemical findings as well as the clinicopathological data relative to the 30 analyzed renal tumors are shown in Table I. A variable NGAL immunoexpression was found in 28/30 cases within the cytoplasm of neoplastic cells (Fig. 1). A membranous staining was also evident in 14/16 clear cell RCCs (Fig. 1a). The peritoneal metastasis of case no. 8 showed a high NGAL immunoexpression (ID score: 8 ) similarly to the primary renal tumor.

With reference to the histotype of analyzed tumors, all papillary and chromophobe RCCs were characterized by high NGAL immunohistochemical expression (ID score: $\geq 4$ ), whereas low expression of this protein was evidenced in all the oncocytomas and urothelial carcinomas. Statistical analyses confirmed that high NGAL expression was significantly associated with papillary and chromophobe histotype of $\mathrm{RCC}(\mathrm{P}=0.016)$ and showed also its significant correlation with higher Fuhrman grade in clear cell and papillary RCCs $(\mathrm{P}=0.004)$ (Table II). No significant correlations were found between NGAL expression and the age, gender or serum iron level of the patients or the size and T stage of the neoplasms (Table II).

\section{Discussion}

NGAL has been recently demonstrated to be involved in human cancer development; accordingly, a high expression of this protein has been encountered in several types of human tumors $(12,17,20)$. Although the renal proximal tubule represents a major source for NGAL synthesis $(25,26,32)$, NGAL expression in renal carcinoma was evaluated only in one study previously (24). The authors reported a weak or absent immunohistochemical staining for this protein in neoplastic kidney; they did not mention the number and histotype of the tumors submitted to the immunohistochemical procedures (24). Therefore, in the present study, we aimed to analyze whether NGAL is expressed in different types of renal tumors such as clear cell, papillary and chromophobe RCCs, or in oncocytomas and urotelial carcinomas.

All types of renal neoplasias were found to express NGAL, as did their normal counterparts. With reference to papillary RCCs, it has been recently suggested that these neoplasias originate from nephrogenic rests within the renal parenchyma (33) and the nephrogenic progenitor cells have also been demonstrated to express NGAL during embryonic kidney development (34).

Intriguingly, a different pattern of labelling was found in positive clear cell RCCs in comparison to the other neoplasias. Apart from the immunoreaction at the cytoplasm of the neoplastic cells, a membranous staining was evident in most of these tumors. We may hypothesize that localization at the plasma membrane of the neoplastic cells may indicate that NGAL is bound to its membrane receptors and not yet internalized. Nonetheless, whether its sub-cellular localization corresponds to a different functional status of NGAL warrants further investigation.

When the level of NGAL expression, reflected by the ID score, was considered in the tumors of our cohort, we noted
Table II. Statistical correlations between NGAL immunoexpression and clinicopathogical parameters.

\begin{tabular}{|c|c|c|c|}
\hline \multirow[b]{2}{*}{ Parameter } & \multicolumn{2}{|c|}{ NAGL } & \multirow[b]{2}{*}{$\mathrm{P}$-value } \\
\hline & Low & High & \\
\hline \multicolumn{4}{|l|}{ Gender } \\
\hline M & 8 & 8 & \\
\hline $\mathrm{F}$ & 6 & 8 & 0.730 \\
\hline \multicolumn{4}{|l|}{ Age } \\
\hline$\leq 60$ years & 6 & 8 & \\
\hline$>60$ years & 8 & 8 & 0.730 \\
\hline \multicolumn{4}{|l|}{ Size } \\
\hline$\leq 6 \mathrm{~cm}$ & 9 & 12 & \\
\hline$>6 \mathrm{~cm}$ & 5 & 4 & 0.694 \\
\hline \multicolumn{4}{|l|}{ Stage } \\
\hline $1-2$ & 11 & 12 & \\
\hline $3-4$ & 3 & 4 & 1.000 \\
\hline \multicolumn{4}{|l|}{ Histotype } \\
\hline Clear cell & 10 & 8 & \\
\hline Urothelial & 2 & 0 & \\
\hline Oncocytoma & 2 & 0 & \\
\hline Chromophobe & 0 & 3 & \\
\hline Papillary & 0 & 5 & 0.016 \\
\hline \multicolumn{4}{|l|}{ Grade } \\
\hline G1 & 7 & 1 & \\
\hline $\mathrm{G} 2$ & 3 & 6 & \\
\hline G3 & 0 & 6 & 0.004 \\
\hline \multicolumn{4}{|l|}{ Serum iron } \\
\hline Low & 5 & 8 & \\
\hline High & 9 & 8 & 0.48 \\
\hline
\end{tabular}

$\mathrm{F}$, female; $\mathrm{M}$, male.

that all papillary and chromophobe RCCs were characterized by high NGAL immunoexpression, whereas oncocytomas and urothelial carcinomas all displayed a low expression of this protein. Then the presence of a significant association between NGAL levels and the histotype of renal tumors was confirmed by the statistical analysis. Since NGAL is involved in iron uptake, we may hypothesize that higher NGAL expression may reflect augmented iron requirement in some RCCs in comparison to others. That papillary and chromophobe histotypes may need higher iron uptake is also suggested by the higher expression of other iron-related proteins, such as lactoferrin (Lf), in these tumors in comparison to other renal neoplasias $(35,36)$. Besides, increased iron transport to the inner cells may be at the basis of carcinogenesis of renal tumors. Indeed, experimental data indicate that renal cell carcinoma is produced by intraperitoneal injection of iron chelates (37); moreover, in a recent review, the role of iron overload in carcinogenesis, through the induction of oxidative DNA damage, has been emphasized (38). 
In our study, high NGAL ID scores were significantly associated with higher histological grade of clear cell and papillary RCCs, in line to that observed in other malignancies, such as thyroid and breast carcinomas, where NGAL levels increase is parallel to the histological grade of the tumors $(12,14,18)$. We may suppose that high grade tumors need higher iron uptake in order to support their increased proliferative potential. It is known that iron depletion leads to cell cycle arrest and apoptosis (39).

The correlation between NGAL immunohistochemical expression and the histotype and grade of renal tumors might have potential therapeutic and diagnostic utility. Tumors characterized by higher NGAL content, such as the chromophobe and papillary RCCs, may be better candidates for anti-cancer agents working as iron chelators (39). Moreover, since NGAL is detectable in the serum and urine samples of subjects affected by NGAL expressing tumors at levels corresponding to those revealed in their neoplastic tissues $(40,41)$, urinary or serum NGAL detection might be the hallmark of metastatic disease in patients operated of renal cancer. Indeed, we found that NGAL expression was retained in the peritoneal metastasis of a clear cell RCC of our cohort.

In conclusion, in the present study we demonstrated for the first time that NGAL is expressed in several histotypes of renal tumors, though at different levels. The highest expression of this protein in papillary and chromophobe RCCs may reflect a higher need of iron uptake, which might be exploited in therapeutic strategies against these tumors through the use of iron-chelators as anti-cancer agents.

If future studies confirm a correlation between NGAL expression in renal tumors and the urinary or blood levels of this protein, the determination of serum or urinary NGAL level might be used for early diagnoses of renal tumor type and grade as well as an indicator of metastatic disease in the follow-up of the patients operated for renal carcinoma.

\section{References}

1. Cowland JB and Borregaard N: Molecular charcterization and pattern of tissue expression of the gene for neutrophil gelatinaseassociated lipocalin from humans. Genomics 45: 17-23, 1997.

2. Bratt T: Lipocalins and cancer. Biochim Biophys Acta 1482: 318-326, 2000.

3. Yang J, Goetz D, Li JY, Wang W, Mori K and Setlik D: An iron delivery pathway mediated by a lipocalin. Mol Cell 10: 1045-1056, 2002.

4. Goetz DH, Willie ST, Arme RS, Bratt T, Borregaard N and Strong RK: Ligand preference inferred from the structure of neutrophil gelatinase associated lipocalin. Biochemistry 39: 1935-1941, 2000.

5. Goetz DH, Holmes MA, Borregaard N, Bluhm ME, Raymond KN and Strong RK: The neutrophil lipocalin NGAL is a bacteriostatic agent that interferes with siderophore-mediated iron acquisition. Mol Cell 10: 1033-1043, 2002.

6. Mori K, Yang J and Barasch J: Ureteric bud controls multiple steps in the conversion of mesenchyme to epithelia. Semin Cell Dev Biol 14: 209-216, 2003.

7. Gwira JA, Wei F and Ishibe S: Expression of neutrophil associated-gelatinase lipocalin regulates epithelial morphogenesis in vitro. J Biol Chem 280: 7875-7882, 2005.

8. Stoesz SP and Gould MN: Overexpression of neu-related lipocalin (NRL) in neu-initiated but not ras or chemically initiated rat mammary carcinomas. Oncogene 11: 2233-2241, 1995.

9. Hannai J, Mammoto T, Seth P, et al: Lipocalin2 diminishes invasiveness and metastasis of Ras-transformed cells. J Biol Chem 280: 3641-3647, 2005.
10. Bolignano D, Donato V, Lacquaniti A, et al: Neutrophil gelatinase-associated lipocalin (NGAL) in human neoplasias: a new protein enters the scene. Cancer Lett (In press).

11. Devireddy LR, Gazin C, Zhu X and Green MR: A cell-surface receptor for lipocalin $24 \mathrm{p} 3$ selectively mediates apoptosis and iron uptake. Cell 23: 1293-1305, 2005.

12. Iannetti A, Pacifico F, Acquaviva R, et al: The neutrophil gelatinase-associated lipocalin (NGAL), a NF-kappaB-regulated gene, is a survival factor for thyroid neoplastic cells. Proc Natl Acad Sci USA 105: 14058-14063, 2008.

13. Yan L, Borregaard N, Kjeldsen L and Moses MA: The high molecular weight urinary matrix metalloproteinase (MMP) activity is a complex of gelatinase B/MMP-9 and neutrophil gelatinase-associated lipocalin (NGAL). Modulation of MMP-9 activity by NGAL. J Biol Chem 276: 37258-37265, 2001.

14. Fernandez CA, Yan L, Louis G, et al: The matrix metalloproteinase-9/nuetrophil gelatinase associated lipocalin complex plays a role in breast tumor growth and is present in the urine of breast cancer patients. Clin Cancer Res 11: 5390-5395, 2005.

15. Lee S, Jilani SM, Nikolova GV, Carpizo D and Iruela-Arispe ML: Processing of VEGF-A by matrix metalloproteinases regulates bioavailability and vascular patterning in tumors. J Cell Biol 169: 681-691, 2005.

16. Devarajan P: Neutrophil gelatinase-associated lipocalin: new paths for an old shuttle. Cancer Ther 5: 463-470, 2007.

17. Stoesz SP, Friedl A, Haag JD, Lindstrom MJ, Clark GM and Gould MN: Heterogeneous expression of the lipocalin NGAL in primary breast cancer. Breast Cancer Res Treat 79: 565-572, 1998.

18. Bauer M, Eickhoff JC, Gould MN, Mundhenke C, Maas N and Friedl A: Neutrophil gelatinase-associated lipocalin (NGAL) is a predictor of poor prognosis in human primary breast cancer. Breast Cancer Res Treat 108: 389-397, 2008.

19. Nielsen BS, Borregaard N, Bundgaard JR, Timshel S, Sehested M and Kjeldsen L: Induction of NGAL synthesis in epithelial cells of human colorectal neoplasia and inflammatory bowel diseases. Gut 38: 414-420, 1996.

20. Zhang XF, Zhang Y, Zhang XH, et al: Clinical significance of Neutrophil gelatinase-associated lipocalin (NGAL) expression in primary rectal cencer. BMC Cancer 9: 134, 2009.

21. Kubben FJ, Sier CF, Hawinkels LJ, et al: Clinical evidence for a protective role of lipocalin-2 against MMP-9 autodegradation and the impact for gastric cancer. Eur J Cancer 43: 1869-1876, 2007.

22. Zhang H, Xu L, Xiao D, et al: Upregulation of neutrophil gelatinase-associated lipocalin in oesophageal squamous cell carcinoma: significant correlation with cell differentiation and tumour invasion. J Clin Pathol 60: 555-561, 2007.

23. Laurell H, Bouisson M, Berthelemy $\mathrm{P}$, et al: Identification of biomarkers of human pancreatic adenocarcinomas by expression profiling and validation with gene expression analysis in endoscopic ultrasound-guided fine needle aspiration samples. World J Gastroenterol 12: 3344-3351, 2006.

24. Friedl A, Stoesz SP, Buckley P and Gould MN: Neutrophil gelatinase-associated lipocalin in normal and neoplastic human tissues. Cell type-specific pattern of expression. Histochem J 31: 433-441, 1999.

25. Bolignano D, Coppolino G, Lacquaniti A, Nicocia G and Buemi M: Pathological and prognostic value of urinary neutrophil gelatinase-associated lipocalin (NGAL) in macroproteinuric patients with worsening renal function. Kidney Blood Press Res 31: 274-279, 2008.

26. Bolignano D, Lacquaniti A, Coppolino G, et al: Neutrophil gelatinase-associated lipocalin (NGAL) and progression of chronic kidney disease. Clin J Am Soc Nephrol 4: 337-344, 2009.

27. Kovacs G, Akhtar M, Beckwith BJ, et al: The Heidelberg classification of renal cell tumours. J Pathol 183: 131-133, 1997.

28. Fuhrman SA, Lasky LC and Limas C: Prognostic significance of morphologic parameters in renal cell carcinoma Am J Surg Pathol 6: 655-633, 1982

29. Guinan P, Sobin LH, Algaba F, et al: TNM staging of renal cell carcinoma: Workgroup No. 3. Union International Contre le Cancer (UICC) and the American Joint Committee on Cancer (AJCC). Cancer 80: 992-993, 1997.

30. Devarajan P, Mishra J, Supavekin S, Patterson LT and Potter S: Gene expression in early ischemic renal injury: clues towards pathogenesis, biomarker discovery, and novel therapeutics. Mol Genet Metab 80: 365-376, 2003. 
31. Moniaux N, Chakraborty S, Yalniz M, et al: Early diagnosis of pancreatic cancer: neutrophil gelatinase-associated lipocalin as a marker of pancreatic intraepithelial neoplasia. Br J Cancer 98: 1540-1547, 2008.

32. Bolignano D, Donato V, Coppolino G, et al: Neutrophil gelatinase-associated lipocalin (NGAL) as a marker of kidney damage. Am J Kidney Dis 52: 595-605, 2008.

33. Szponar A, Zubakov D, Pawlak J, Jauch A and Kovacs G: Three genetic developmental stages of papillary renal cell tumors: duplication of chromosome 1q marks fatal progression. Int J Cancer 124: 2071-2076, 2009.

34. Yang J, Mori K, Li JY and Barasch J: Iron, lipocalin and kidney epithelia. Am J Physiol Renal Physiol 285: F9-F18, 2003.

35. Giuffrè G, Barresi V, Skliros C, Barresi G and Tuccari G Immunoexpression of lactoferrin in human sporadic renal cell carcinomas. Oncol Rep 17: 1021-1026, 2007.

36. Loughlin KR, Gittes RF, Partridge D and Stelos P: The relationship of lactoferrin to the anemia of renal cell carcinoma. Cancer 59: 566-571, 1987.
37. Liu M and Okada S: Induction of free radicals and tumors in kidneys of Wistar rats by ferric ethylenediamine-N,N-diacetate. Carcinogenesis 15: 2817-2821, 1994.

38. Toyokuni S: Role of iron in carcinogenesis: Cancer as a ferrotoxic disease. Cancer Sci 100: 9-16, 2009.

39. Le NT and Richardson DR: The role of iron in cell cycle progression and the proliferation of neoplastic cells. Biochim Biophys Acta 1603: 31-46, 2002.

40. Lim R, Ahmed N, Borregaard N, et al: Neutrophil gelatinaseassociated lipocalin (NGAL) an early screening biomarker for ovarian cancer: NGAL is associated with epidermal growth factor-induced epithelio-mesenchymal transition. Int J Cancer 120: 2426-2434, 2007.

41. Smith ER, Zurakowski D, Saad A, Scott RM and Moses MA: Urinary biomarkers predict brain tumor presence and response to therapy. Clin Cancer Res 14: 2378-2386, 2008. 